\title{
Foundation of Graduate Study in Mechanical Engineering-Advanced Dynamics
}

\author{
Shuh Jing Ying \\ Department of Mechanical Engineering, University of South Florida, Tampa, FL 33620, USA
}

Received: February 15, 2016 / Accepted: March 01, 2016 / Published: April 30, 2016.

\begin{abstract}
Dynamics is the foundation of undergraduate study in mechanical engineering. If you are good in dynamics, you will be good on all other courses. Advanced dynamics is the foundation for graduate study. The author taught advanced dynamics for more than ten years, this is the author's observation. Why it is so? Because the course of advanced dynamics covers usually many mathematical fundamentals such as vectors, tensors, matrices and rotation operators; principles and applications in dynamics from particle dynamics to rigid body motion, from small oscillation to vibration of systems with multiple degrees of freedom, the author's course covers also special relativity theory. They are very innovative. And they set the foundation for the study of all the graduate courses. Science is always in progress, dynamics is in the same form. Just say a few examples to illustrate the frontier of dynamics: missile shooting missile is important in our defense, the author covered this as an example in particle dynamics. Space ship travels from Earth to Mars is another example. Several rotational motions with different axes can be combined to one through the use of rotation operator. This is important because it usually can save time. All these examples will be included in this paper in some details.
\end{abstract}

Key words: Advanced dynamics, graduate study, mechanical engineering.

\section{Introduction}

This paper was originally prepared for the Third International Conference and Exhibition of Mechanical and Aerospace Engineering in San Francisco, USA in 2015. When the author was invited as a speaker for the conference, the author thinks he must choose a topic that is interesting to general public and also the author is very familiar to it myself. Because the author published a book in Advanced Dynamics, so the author chooses this as his topic to talk.

Because the knowledge of vector is needed immediately in dynamics, so the author arranged vector algebra and vector analyses in the first chapter of the book [1]. Other mathematical topics, such as matrices, tensors, dyadics, and rotation operators, are arranged in Chapter 6. Chapter 6 is entirely mathematical, so that engineering students are exposed to more applied mathematics. Some applications are

Corresponding author: Shuh Jing Ying, professor emeritus, research field: rehabilitation engineering. included with each subject to make them easily understandable and more interesting. Engineers wishing to extend their knowledge through journal papers should pay special attention to this chapter.

The special relativity theory is covered in Chapter 10. It is arranged here to specially broaden readers' mind. The time and space coordinates are related such that for one person travelling near speed of light, just a few days for this person can be many years to a person in a stationary system. This is proved to be true scientifically. Moreover one also can prove that, an event in the past could be observed as a present in another system. Readers are urged to consider that, just as space and time are now interrelated through the relativity theory, new developments may one day modify our thoughts concerning our most basic scientific concepts and principles.

\section{Missile Shooting Missile in Midair}

Study of the collision of two missiles in midair is based on the motions of individual missiles. To simplify 
the problem let us model them as particles. The subject is studied in three steps. First is the motion of a single particle in air, then is the transfer of coordinates, finally is the collision of two particles in the midair.

\subsection{The Motion of a Single Particle in Air}

Consider a missile moving in air as a particle with a mass decreasing constantly. The thrust applied is constant in magnitude and always in the direction of particle's velocity. The coordinates are chosen such that, the $\mathrm{x}-\mathrm{z}$ plane contains the trajectory with $\mathrm{z}$ axis perpendicular to the ground. The trajectories for different thrusts can be found as follows. In this example, three different thrusts are assumed to be $F=$ $14,500,15,000$ and $15,500 \mathrm{~N}$. The initial conditions of the missile are $m_{0}=1,000 \mathrm{~kg}$ and $v_{0}=150 \mathrm{~m} / \mathrm{s}$ at an angle of $80 \mathrm{deg}$ with the $\mathrm{x}$ axis. The mass decreasing rate is $\dot{m}=3 \mathrm{~kg} / \mathrm{s}$. The governing equations are given below:

$$
\begin{aligned}
m \frac{\mathrm{d} v_{x}}{\mathrm{~d} t} & =F \frac{v_{x}}{\sqrt{v_{x}^{2}+v_{z}^{2}}} \\
m \frac{\mathrm{d} v_{z}}{\mathrm{~d} t} & =F \frac{v_{z}}{\sqrt{v_{x}^{2}+v_{z}^{2}}}-m g \\
m & =m_{0}-\dot{m} t \\
\frac{\mathrm{d} x}{\mathrm{~d} t} & =v_{x} \\
\frac{\mathrm{d} z}{\mathrm{~d} t} & =v_{z}
\end{aligned}
$$

Eqs. (1) and (2) are nonlinear and cannot be solved analytically. However, they can be integrated numerically by the Runge-Kutta method. The trajectory then can be obtained from Eqs. (4) and (5). Three trajectories are obtained for the three different values of thrust. The results are given in Fig. 1. In the numerical integration, the increment of time used is $0.01 \mathrm{~s}$ and the total duration is more than $160 \mathrm{~s}$. A convergence check is performed before the results are calculated.

\subsection{Conversion of Coordinates}

Before studying the collision of two missiles in the next subsection, we need to discuss the conversion of coordinates. Because two missile sites are a few hundred kilometers apart, each missile may be described by its own coordinate system as given in previous subsection, and then they must be converted into one set of coordinates. The procedure of establishing the relationship between the two sets of coordinates is referred to as the conversion of coordinates.

Consider that, the coordinate system XYZ is to exist permanently and the coordinate system xyz is to be converted. The xyz axes are parallel to XYZ axes initially. The origin of xyz coordinates in XYZ system is $X_{0}, Y_{0}, Z_{0}$. The xyz is rotated with respect to y axis by an angle of $\Phi$ and then rotated with respect to rotated $z$ axis by an angle of $\theta$. The relative location between

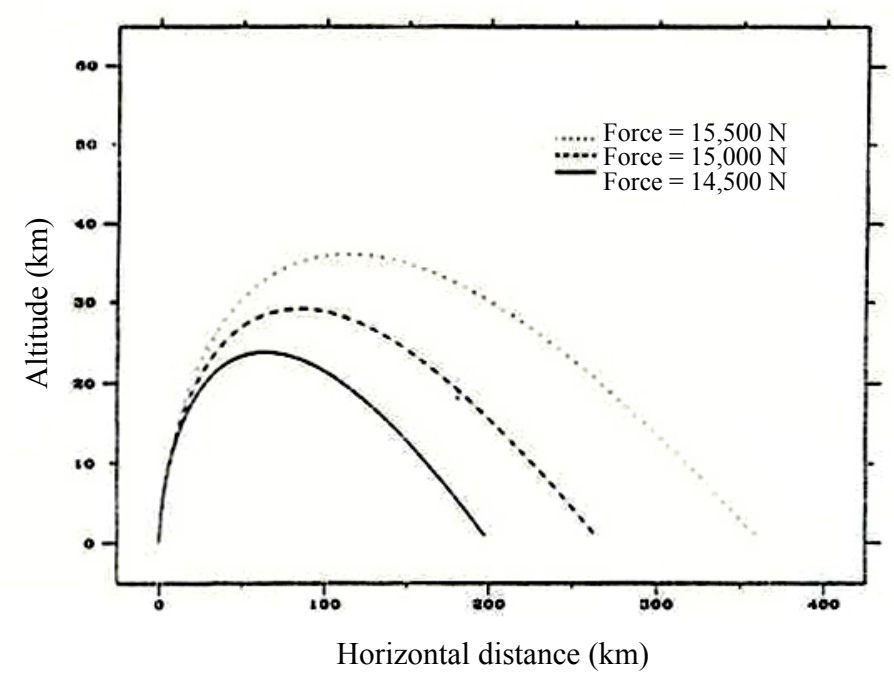

Fig. 1 The trajectories of the missile. 


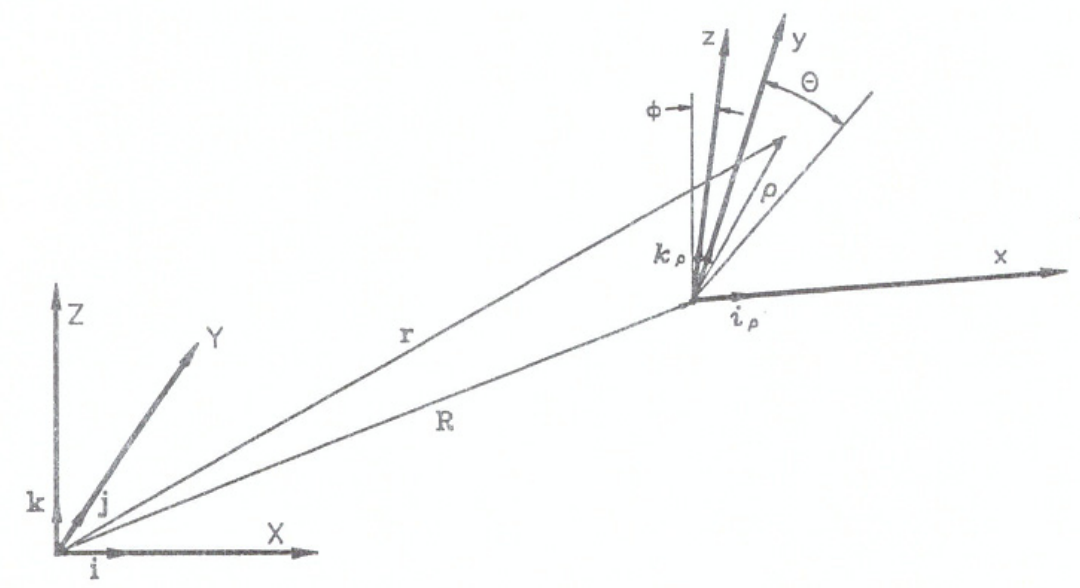

Fig. 2 Relationship between XYZ system and xyz system.

the two systems is given in Fig. 2. Omit the detail derivation, the equations for the conversion are given below:

$$
\begin{gathered}
X=X_{0}+x \cos \theta \cos \Phi-y \sin \theta \cos \Phi+z \sin \Phi \\
Y=Y_{0}+x \sin \theta+y \cos \theta \\
Z=Z_{0}-x \cos \theta \sin \Phi+y \sin \theta \sin \Phi+\mathrm{z} \cos \Phi
\end{gathered}
$$

In a special case, if there is no rotation with respect to the y axis, i.e., $\Phi=0$, Eqs. (6)-(8) reduce to:

$$
\begin{gathered}
X=X_{0}+x \cos \theta-y \sin \theta \\
Y=Y_{0}+x \sin \theta+y \cos \theta \\
Z=Z_{0}+z
\end{gathered}
$$

This set of equations is referred to as flat ground conversion.

On the other hand, when two coordinate systems are apart by an order of a few hundred kilometers on the surface of the Earth, the effect of the spherical surface must be taken into consideration. Consider that, the coordinate systems are on the spherical surface of the Earth as shown in Fig. 3. The XYZ system is so chosen that, the plan containing $\mathrm{X}$ and $\mathrm{Z}$ axes is the same plan containing $R_{0}, R_{1}$, and $R$. The unit vector $k$ is along the vector $R_{0}$, that is pointing from the center of Earth radially to the origin of XYZ. $R_{1}$ is the position vector of the origin of xyz. Again omitting the detail derivation the equations for the conversion are given below.

$$
\begin{gathered}
X=R_{0} \sin \Phi+x \cos \theta \cos \Phi- \\
y \sin \theta \cos \Phi+z \sin \Phi \\
Y=x \sin \theta+y \cos \theta \\
Z=-R_{0}(1-\cos \Phi)-x \cos \theta \sin \Phi+ \\
y \sin \theta \sin \Phi+z \cos \Phi
\end{gathered}
$$

This set of equations is known as the conversion of coordinates on spherical ground.

\subsection{Collision of Missiles in Midair}

Suppose that, a missile is launched from the enemy side, which is designated as the first missile. Through the detection by a satellite, the trajectory can be simulated as given in Subsection 2.1 with thrust of $F=$ $14,500 \mathrm{~N}$. The coordinates are transferred. Because of the action taken for the determination of the trajectory of the first missile, the time for launching the second

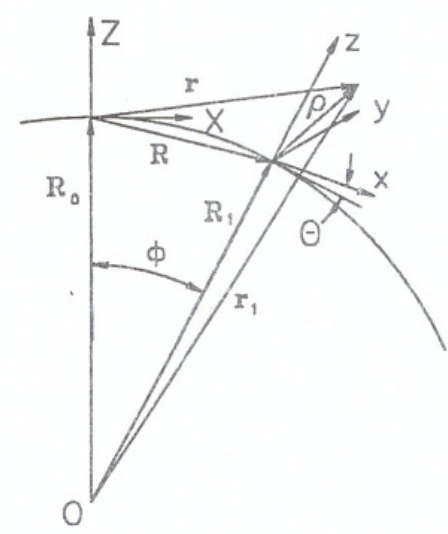

Fig. 3 Transfer of coordinates on spherical surface. 
missile is delayed by $60 \mathrm{~s}$. To simplify the calculation, the trajectories of the two missiles are assumed to be contained in the same plane, but the launching sites are $200 \mathrm{~km}$ apart. The data for the second missile are given as follows: initial mass $m_{0}=1,000 \mathrm{~kg}$, thrust $F=$ $16,000 \mathrm{~N}$, initial velocity $=300 \mathrm{~m} / \mathrm{s}$, and the mass decreasing rate $=3 \mathrm{~kg} / \mathrm{s}$. We want to find the launching angle of the second missile so that the two missiles are to collide high above the ground. The conversion of coordinates is treated in two different ways: (1) flat ground and (2) spherical ground.

(1) Consider that, the two launching sites are on flat ground. Each missile is governed by the equations as given in Eqs. (1)-(5). Equations are nonlinear and are solved by numerical integration with the Runge-Kutta method. The conditions used for the first missile are:

$$
\begin{gathered}
\left(m_{1}\right)_{0}=1,000 \mathrm{~kg} \\
\dot{m}_{1}=3 \mathrm{~kg} / \mathrm{s} \\
\left(v_{1}\right)_{0}=150 \mathrm{~m} / \mathrm{s} \\
\alpha_{1}=80 \mathrm{deg} \\
F_{1}=14,500 \mathrm{~N}
\end{gathered}
$$

where, $\alpha$ is the launching angle measured from $\mathrm{x}$ axis. The coordinates are transferred simple by:

$$
\begin{gathered}
\mathrm{X}_{1}=X_{0}-x_{1} \\
Z_{1}=z_{1}
\end{gathered}
$$

The conditions used for the second missile are

$$
\begin{gathered}
\left(m_{2}\right)_{0}=1,000 \mathrm{~kg} \\
\dot{m}_{2}=3 \mathrm{~kg} / \mathrm{s} \\
\left(v_{2}\right)_{0}=300 \mathrm{~m} / \mathrm{s} \\
F_{2}=16,000 \mathrm{~N}
\end{gathered}
$$

The launching angle of the second missile is determined with a trial and error method performed on computer. In the calculation, the first number used is $1.00 \mathrm{rad}$ with the increment of \pm 0.01 . To detect whether the collision is going to take place or not, the distance between two missiles is calculated. The unsuccessful simulation terminates as the distance between them increases. When the collision is nearly occurring, finer increments for the launching angle and the time step are used. For the present study, the increments for the final step are $\Delta \alpha=2.0 \mathrm{E}-7$ and $\Delta t=5.0 \mathrm{E}-5 \mathrm{~s}$. The collision condition is reached when the distance between two missiles is less than $8 \mathrm{~cm}$. The launching angle of the second missile is found to be $0.9821454 \mathrm{rad}$. The collision is taking place at $144.8327 \mathrm{~s}$ after the launching of the first missile and is $84.8327 \mathrm{~s}$ after the launching of the second missile. The coordinates at the collision are $X=66.82 \mathrm{~km}, Z=16.26 \mathrm{~km}$.

(2) For a spherical surface, the equations governing the motions of missiles are the same as those used in part (1). Because the trajectories of the missiles are assumed to be in the same plane, the coordinates of the first missile are transferred using Eqs. (12) and (14) with $y=0$. These equations are as follows:

$$
\begin{gathered}
X=R_{0} \sin \Phi+x \cos \theta \cos \Phi+z \sin \Phi \\
Z=-R_{0}(1-\cos \Phi)-x \cos \theta \sin \Phi+z \cos \Phi
\end{gathered}
$$

For the present case $R_{0}=6,371.23 \mathrm{~km}, \theta=\pi$ and $\Phi$ $=0.031391112$. Substituting these values into above equations, we have

$$
\begin{array}{r}
X=199,967.155-0.99950734 x+0.003138596 z(\mathrm{~m}) \\
Z=-3138.8535+0.03138596 x+0.99950734 z(\mathrm{~m})
\end{array}
$$

The calculation procedure is the same as that used in part (1). The launching angle for the second missile is determined to be $0.9929676 \mathrm{rad}$. and the collision occurs at $145.1400 \mathrm{~s}$ after the launching of the first missile and $85.1400 \mathrm{~s}$ after launching of the second missile. It is important to point out that, the missiles will not collide if $\alpha$ is set at $0.9821454 \mathrm{rad}$. because the Earth's surface is actually spherical. The coordinates at the collision are $X=66.64 \mathrm{~km}$ and $Z=17.18 \mathrm{~km}$. The missile trajectories are shown in Fig. 4.

\section{Interplanetary Trajectories}

This subject is arranged at the end of Chapter 5 in the author's book of Advanced Dynamics [2]. That means students have the basic knowledge in particle dynamics. In this chapter, the author first talked about single stage rocket, multistage rocket, and then motion of a particle in central force field. The author also reviewed a part of analytical geometry for conic curves. So students are well prepared for studying this subject. 


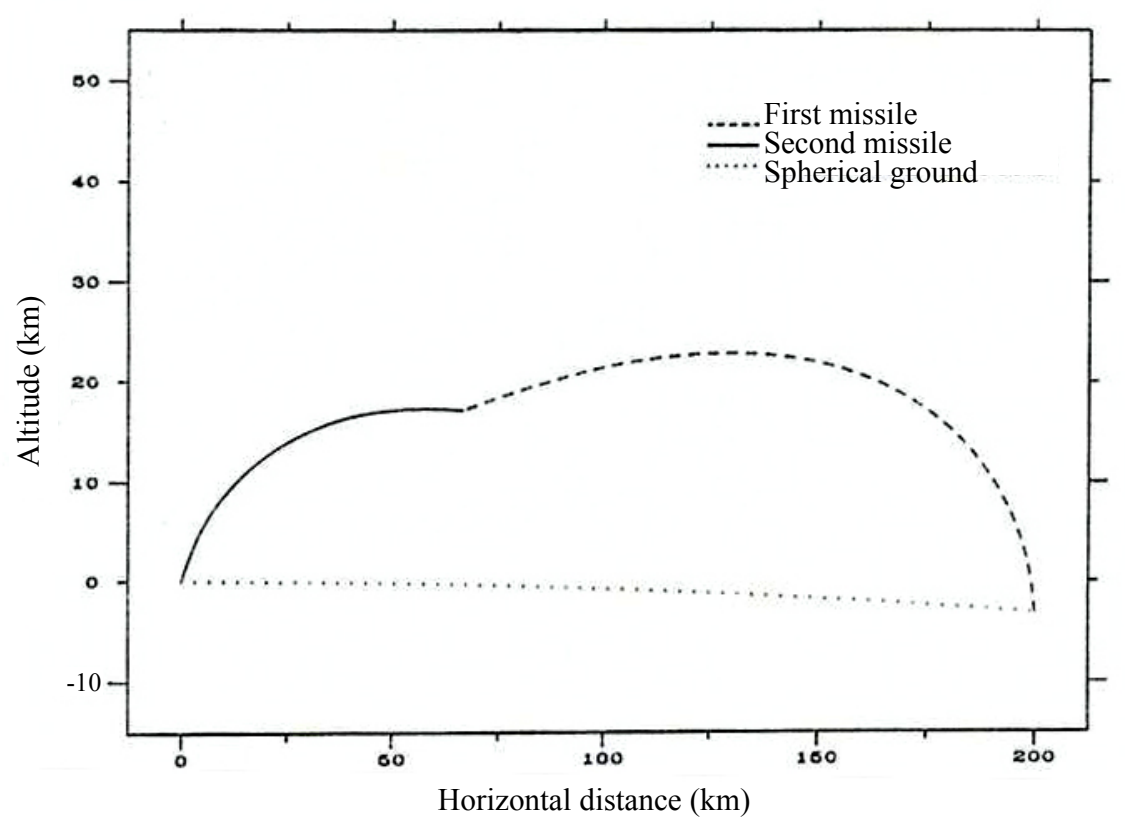

Fig. 4 Missile to missile trajectories on spherical surface.

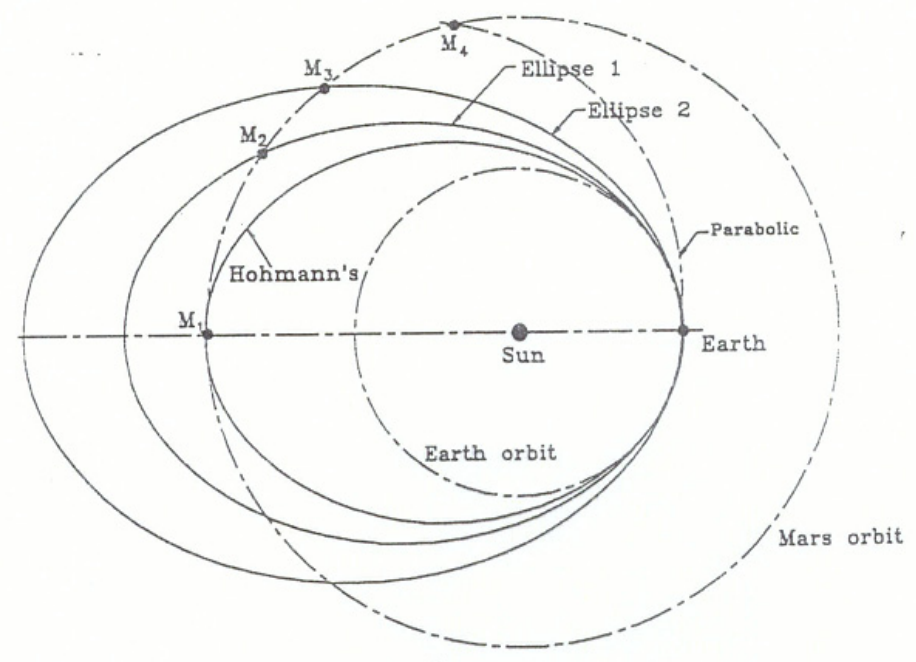

Fig. 5 Four different trajectories for Earth-Mars journey.

Table 1 Characteristics of different trajectories.

\begin{tabular}{lllll}
\hline Name of trajectory & Eccentricity, $\varepsilon$ & $U_{\text {Oberth }}(\mathrm{km} / \mathrm{s})$ & $\Delta U_{\text {Mars }}(\mathrm{km} / \mathrm{s})$ & Time required (days) \\
\hline Hohmann & 0.2075 & 11.6 & 2.6 & 256 \\
Ellipse 1 & 0.2525 & 11.7 & -2.6 & 175 \\
Ellipse 2 & 0.3418 & 12.1 & -5.0 & 135 \\
Parabolic & 1.0 & 16.7 & -16.9 & 70 \\
\hline
\end{tabular}

Suppose that, we send a space probe from Earth to Mars. There are different trajectories we can choose as shown in Fig. 5. The characteristics of the different trajectories are given in Table 1.

Where $U_{\text {Oberth }}$ is the velocity required for the space probe leaving Earth to get into the transfer orbit, that is the orbit from Earth orbit to Mars orbit. $\Delta U_{\text {Mars }}$ is the increment in velocity from transfer orbit to Mars orbit. Time required is the time for the space probe travelling from Earth to Mars. 


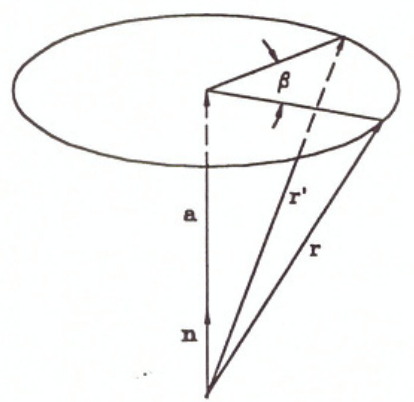

(a)

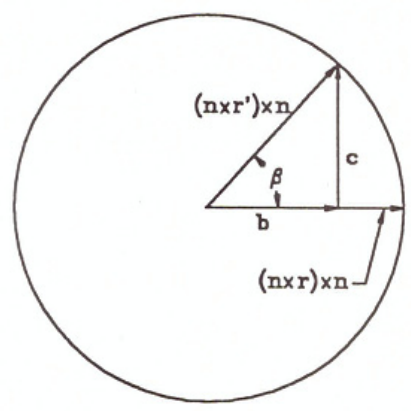

(b)

Fig. 6 Rotation of $r$ about $n$.

Detail calculations are given in the example in the author's book [3] for ellipse 1. Ellipse 2 and parabolic trajectories are assigned as home work problems in the book.

\section{Combined Rotation}

In Section 2, the author talked about the collision of missiles in midair. In it, the delay time for the launching of the second missile is assumed to be $60 \mathrm{~s}$. This interval includes the time to rotate the launching equipment to a proper angle. Certainly this operation could be done by using Euler angles, but that approach takes too much time. With the operation given in Section 2, we will find that, the operation is simplified and saves time.

Consider that, a position vector $r$ is rotated with respect to vector $n$ by angle $\beta$. The geometry of this operation is shown in Fig. 6. The operation can be expressed by the following equation:

$$
r^{\prime}=\overleftrightarrow{R}(n, \beta) \cdot r
$$

where, $\overleftrightarrow{R}$ is defined as a rotation operator and can be expressed as:

$\overleftrightarrow{R}(n, \beta)=(1-\cos \beta) n n+\cos \beta \overleftrightarrow{1}+\sin \beta(n \times \overleftrightarrow{1})$

Dyadic notation is used in above equation. $\overleftrightarrow{1}$ is a unit dyadic.

Suppose a rigid body to be rotated by two steps. First it is rotated about the $k$ ' axis by an angle of $\Phi$ and then it is rotated about the $k$ axis by an angle of $\Psi$. These two rotations can be combined into one rotation. The rotation axis $n$ and the angle of the combined rotation $\beta$ can be found in the following equations.

$$
\begin{gathered}
n=\frac{1}{\sin (\beta / 2)}\left[\cos \frac{\Psi}{2} \sin \frac{\Phi}{2} k+\sin \frac{\Psi}{2} \cos \frac{\Phi}{2} k^{\prime}+\right. \\
\left.\sin \frac{\Psi}{2} \sin \frac{\Phi}{2}\left(k^{\prime} \times k\right)\right] \\
\cos \frac{\beta}{2}=\cos \frac{\Psi}{2} \cos \frac{\Phi}{2}-\sin \frac{\Psi}{2} \sin \frac{\Phi}{2} \cos \theta
\end{gathered}
$$

where, $\theta$ is the angle between $k$ and $k^{\prime}$. To illustrate the use of above two equations, there is an example in the book of Advanced Dynamics [4], but it is omitted here. Certainly the equations are valid.

\section{Conclusions}

It is easily seen that, dynamics is progressing just as other sciences. And it is true that, advanced dynamics is the foundation for graduate study in mechanical engineering. Graduate students should put a great effort on this course to set a good foundation for other courses.

\section{References}

[1] Ying, S. J. 1997. Advanced Dynamics. Virginia, USA: American Institute of Aeronautics and Astronautics.

[2] Chobotov, V. A., ed. 2002. Orbital Mechanics. Virginia, USA: American Institute of Aeronautics and Astronautics.

[3] Brown, C. D. 1996. Spacecraft Propulsion. Washington, D.C., USA: American Institute of Aeronautics and Astronautics.

[4] Greenwood, D. T. 2003. Advanced Dynamics. Cambridge, UK: Cambridge University Press. 